hep-ph/9801262

$\mathrm{PSU} / \mathrm{TH} / 192$

\title{
Proof of Factorization for Deeply Virtual Compton Scattering in QCD
}

\author{
John C. Collins and Andreas Freund \\ Department of Physics, Penn State University, 104 Davey Lab., University Park, PA 16802, \\ U.S.A.
}

(2 July 1998)

\begin{abstract}
We show that factorization holds for the deeply virtual Compton scattering amplitude in QCD, up to power suppressed terms, to all orders in perturbation theory. The theorem applies to the production of off-shell photons as well as real photons. We give a detailed treatment of the situation where one of the two partons joining the parton density to the hard scattering has zero longitudinal momentum.
\end{abstract}

Keywords: Factorization, deeply virtual Compton scattering

11.10.Jj, 12.38.Aw, 12.38.Bx, 13.60.Fz

Typeset using REVTEX 


\section{INTRODUCTION}

In this paper, we prove factorization for the deeply virtual Compton scattering (DVCS) amplitude in QCD up to power suppressed terms, to all orders in perturbation theory. This proof is important because of the recent great interest in DVCS [1 10]. One important use of DVCS is as a probe of off-forward (or nondiagonal) distributions [1, 3, 11, 13]. These differ from the usual parton distributions probed in inclusive reactions by having a non-zero momentum transfer between the proton in the initial and final state.

A related process which is also used to probe off-diagonal parton densities is exclusive meson production in deep-inelastic scattering [14, 15], for which a proof of factorization was given in 12]. Compared with this process, DVCS is simpler because the composite meson in the final state is replaced by an elementary particle, the photon, and thus there is no meson wave function in the factorization formula.

In the case of a scalar field theory, Anikin and Zavialov [16] proved a non-local operator product expansion, from which follows the factorization theorem for DVCS, as shown by Müller et al. [17]. From the point of view of these papers, the new results in the present paper consist of an extension of the results to QCD. However, we do not derive an explicit form of an $R$-operation for the coefficient functions, unlike Anikin and Zavialov.

In a separate line of development, Ji [1] and Radyushkin [2] provided key insights that indicate that a factorization theorem is valid for DVCS, and then Radyushkin provided an all-orders proof in [3]. In this paper we provide an alternative proof, and give a new treatment of some problems that were treated in Ref. [3] but that were perhaps not fully solved. The primary technical difference between Radyushkin's derivations and ours is that he uses the $\alpha$-parametric representation for Feynman graphs, whereas we use the momentum representation, which we consider to be more direct. Our proof follows the general lines of proofs of factorization for other processes given in [18,19], and the most noteworthy feature is that, particularly for the case of production of off-shell photons, the proof is simpler than for any other process. Even for ordinary deep-inelastic scattering one needs to discuss the cancellation of soft gluon exchanges and of final-state interactions, whereas these complications are not present in the leading power for DVCS.

The paper is organized in the following way: After stating the theorem in Sec. II], we show in Sec. [II how the proof given in Ref. [12] is readily adapted to DVCS. The complications mentioned above concern the situation when one of the two lines connecting the parton density to the hard scattering carries zero longitudinal momentum, and these are given a detailed treatment in Sec. IIIH.

\section{FACTORIZATION THEOREM}

The process under consideration is DVCS which is the elastic scattering of virtual photons:

$$
\gamma^{*}(q)+P(p) \rightarrow \gamma^{(*)}\left(q^{\prime}\right)+P^{\prime}(p-\Delta)
$$

where the diffracted proton $P^{\prime}$ may also be replaced by a low-mass excited state and the finalstate photon can be either real or time-like. This process is the hadronic part of $e p \rightarrow e \gamma p^{\prime}$ for a real photon or of $e p \rightarrow e \mu^{+} \mu^{-} p^{\prime}$ for a time-like photon. 
It is convenient to use light-cone coordinates with respect to the collision axis. The momenta in the process then take the form:

$$
\begin{aligned}
p^{\mu} & =\left(p^{+}, \frac{m^{2}}{2 p^{+}}, \mathbf{0}_{\perp}\right) \\
q^{\mu} & \simeq\left(-x p^{+}, \frac{Q^{2}}{2 x p^{+}}, \mathbf{0}_{\perp}\right) \\
q^{\prime \mu} & \simeq\left(x p^{+} \frac{\Delta_{\perp}^{2}+\alpha Q^{2}}{Q^{2}}, \frac{Q^{2}}{2 x p^{+}}, \Delta_{\perp}\right) \\
\Delta^{\mu} & \simeq\left(x(1+\alpha) p^{+},-\frac{\Delta_{\perp}^{2}+m^{2}(1+\alpha) x}{2(1-x-\alpha x) p^{+}}, \Delta_{\perp}\right) .
\end{aligned}
$$

Here, $x$ is the Bjorken scaling variable, $Q^{2}$ is the virtuality of the initial photon, $m^{2}$ is the proton mass, $t=\Delta^{2}$ is the momentum transfer squared, and $\alpha$ is a parameter that specifies the virtuality of the outgoing photon: $q^{2}=\alpha Q^{2}$. Thus, $\alpha=0$ for a real photon and $\alpha>0$ for a time-like photon. Finally, $\simeq$ means "equality up to power suppressed terms".

The theorem to be proved is that the amplitude for the process (1) is:

$$
T=\sum_{i} \int_{-1+x}^{1} d x_{1} f_{i / p}\left(x_{1}, x_{2}, t, \mu\right) H_{i}\left(x_{1} / x, x_{2} / x, \mu\right)+\text { power-suppressed corrections }
$$

where $f_{i / p}$ is a nondiagonal parton distribution and $H_{i}$ is the hard-scattering coefficient for scattering off a parton of type $i$. We let $x_{1}$ be the momentum fraction of parton $i$ coming from the proton, so that $x_{2}=x_{1}-x(1+\alpha)$ is the momentum fraction which is returned to the proton by the other parton line joining the parton distribution and the hard scattering. There is implicit polarization dependence in the amplitude. $\mu$ is the usual renormalization/factorization scale, which should be of order $Q$ to allow calculations of the hard scattering coefficients within finite-order perturbation theory. The $\mu$ dependence of $f_{i / p}$ is given by equations of the DGLAP and Brodsky-Lepage kind [1, 3, 11, 14, 12, 13, . The parton distributions in Eq. (3), together with their evolution equations, are defined using the conventions of [12, 13]. They may easily be transformed into those given in $[1]$ by a change of normalization and of kinematic variables.

\section{PROOF OF THEOREM}

The proof of our theorem Eq. (3) can be summarized as follows ? 2 :

\footnotetext{
${ }^{1}$ We define a vector in light cone coordinates by:

$$
V^{\mu}=\left(V^{+}, V^{-}, V_{\perp}\right)=\left(\frac{V^{0}+V^{3}}{\sqrt{2}}, \frac{V^{0}-V^{3}}{\sqrt{2}}, V^{1,2}\right) .
$$

${ }^{2}$ For a very detailed account of the basic steps and potential problems see Ref. [12].
} 


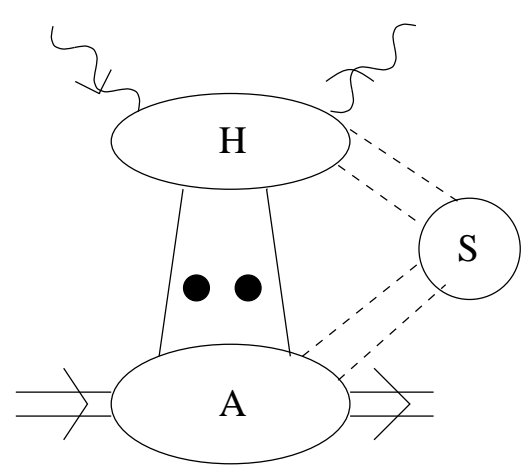

a)

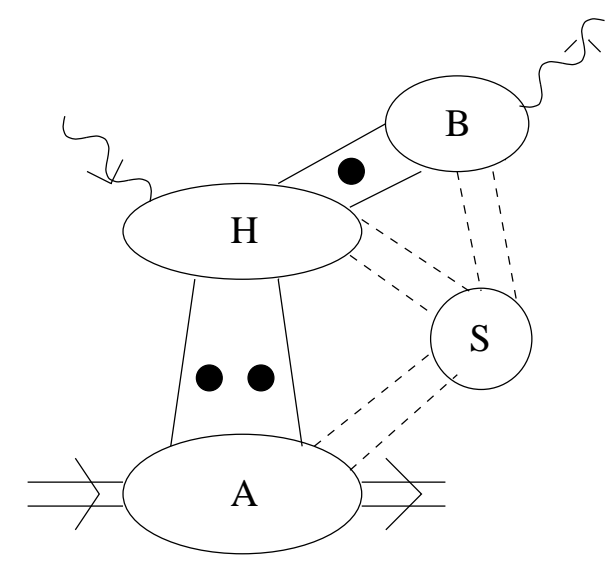

b)

FIG. 1. a) Reduced graph for DVCS with direct coupling for the out-going photon to hard subgraph. b) The same without a direct like coupling for the out-going photon.

- Establish the non-ultra-violet regions in the space of loop momenta contributing to the amplitude.

- Establish and prove a power counting formula for these regions.

- Determine the leading regions of the amplitude.

- Define the necessary subtractions in the amplitude to avoid double counting.

- Taylor expand the amplitude to obtain a factorized form.

- Show that the part containing the long-distance information can be expressed through matrix elements of renormalized, bi-local, gauge invariant operators of twist-2.

\section{A. Regions}

First let us establish the regions in the space of loop momenta contributing to the asymptotics of the amplitude, i.e., the generalized reduced graphs. The steps leading to the generalized reduced graphs are identical to the steps 1-3 in Sec. IV of Ref. [12], i.e., scale all momenta by a factor $Q / m$, use the Coleman-Norton theorem to locate all pinch-singular surfaces in the space of loop momenta (in the zero-mass limit), and finally identify the relevant regions of integration as neighborhoods of these pinch-singular surfaces.

The results are the two kinds of reduced graph shown in Fig. 1. There, $A$ and $B$ denote collinear graphs with one large momentum component in the + and - direction respectively, $H$ denotes the hard scattering graph, and $S$ denotes a graph with all of its lines soft, i.e., in the center-of-mass frame all the components of the momenta in $S$ are much smaller than $Q$. Note that, of the external momenta, $p$ and $p^{\prime}$ belong to $A, q^{\prime}$ belongs to $B$ or $H$, and $q$ belongs to $H$. 


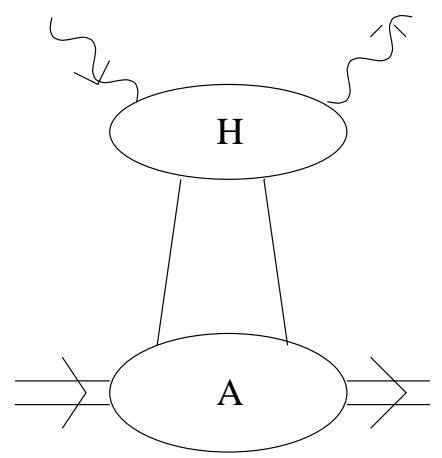

a)

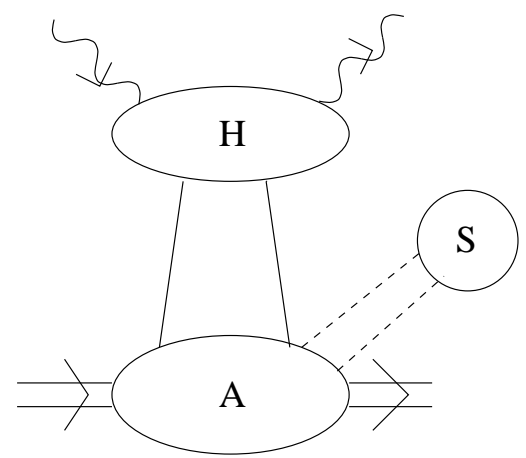

b)

FIG. 2. Those reduced graphs that contribute to the leading regions in DVCS.

When the two external photons have comparably large virtualities, the only reduced graphs are of the first kind, Fig. 17a, where the out-going photon couples directly to the hard scattering. But when the out-going photon has much lower virtuality than the incoming photon, for example, when it is real, we can also have the second kind of reduced graph, Fig. 1 b, where the out-going photon couples to a $B$ subgraph. As we will see later, power counting will show that the second kind of reduced graph, Fig. [ $\mathrm{b}$, is power suppressed compared to the first kind, with a direct photon coupling. This implies that we will avoid all the complications which were encountered in [12] that are associated with the meson wave function.

\section{B. Power Counting}

Each reduced graph codes a region of loop-momentum space, a neighborhood of the surface $\pi$ of a pinch singularity in the zero-mass limit. The contribution to the amplitude from a neighborhood of $\pi$ behaves like $Q^{p(\pi)}$, modulo logarithms, in the large- $Q$ limit, with the power given by

$$
\begin{aligned}
p(\pi)= & 4-n(H)-\#(\text { quarks from } S \text { to } A, B)-3 \#(\text { quarks from } S \text { to } H) \\
& -2 \#(\text { gluons from } S \text { to } H) .
\end{aligned}
$$

where $n(H)$ is the number of collinear quarks, transversely polarized gluons, and external photons attaching to the hard subgraph $H$. Such results were obtained by Libby and Sterman [20,21]. The particular form of Eq. (4) was given in [12 together with a proof that applies without change to DVCS.

The well-known problem of gluons with scalar polarization (see, for example, 18,22,23) will be dealt with later on. Suffice it to say here that gluons with such a polarization can be factorized into the parton distributions by using gauge-invariance arguments. 


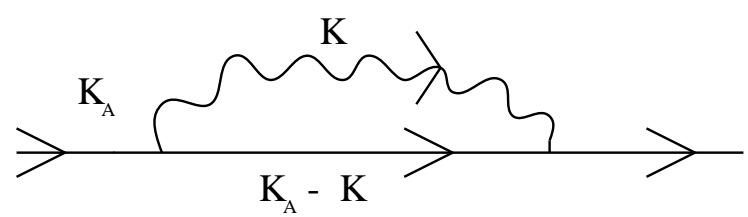

FIG. 3. Soft gluon loop attaching to collinear line.

\section{Leading Regions}

The leading regions for the amplitude are those with the largest exponent $p(\pi)$ in Eq. (四). It is easy to see that these correspond to the reduced graphs in Fig. 2, independently of whether the out-going photon is real or far off-shell. The corresponding power is $Q^{0}$. These reduced graphs have direct photon couplings to the hard subgraph, they have exactly two parton lines connecting the collinear subgraph $A$ to the hard subgraph $H$, and they have no soft lines connecting to $H$. The two kinds of graph differ only by the absence or presence of a soft subgraph that connects to $A$ alone.

Among the other reduced graphs, which are non-leading for our process, are those of the type in Fig. 1 $\mathrm{b}$, which are leading in the case of diffractive meson production, where the leading region gives $Q^{-1}$.

In the case of a photon that is off-shell by order $Q^{2}$, the amplitude for production of the photon behaves like $Q^{0}$, the same as for a real photon. However, the physically observed process includes the decay of the time-like photon (to a $\mu^{+} \mu^{-}$, for example), which results in a power suppression of the observed cross section by $1 / Q^{2}$ compared with the cross section for making real photons.

\section{Proof of absence of a soft part in leading regions}

As mentioned in Sec. IIIC, there might, in principle, be a soft part $S$ in the leading reduced graph connected solely to the $A$ graph by gluons, as shown in Fig. 2p. Note that by Eq. (四), quarks connecting $S$ to $A$ would lead to a power suppression. We will now show that this soft part $S$ is indeed absent, and so we only need to consider regions of the form of Fig. 2a.

We will first examine a simple one loop example, Fig. 3. The external quark is part of the $A$ subgraph in Fig. $2 \mathrm{~b}$, and the gluon is soft. So we parameterize the momenta by:

$$
\begin{aligned}
k_{A} & =\left(x_{1} p^{+}, k_{A}^{-}, \mathbf{k}_{\mathbf{A}, \perp}\right), \\
k & =\left(k^{+}, k^{-}, \mathbf{k}_{\perp}\right) .
\end{aligned}
$$

where the $k_{A}^{+}$is $O(Q)$ and all the other components are of $O(m)$ or smaller.

If we omit irrelevant factors in the numerator, the loop integral takes the following form:

$$
\int_{\text {soft } k} d^{4} k \frac{1}{\left(k^{2}+i \epsilon\right)\left[\left(k_{A}-k\right)^{2}-m^{2}+i \epsilon\right]}
$$




$$
\begin{aligned}
& =\int_{\text {soft } k} d^{4} k \frac{1}{\left(2 k^{+} k^{-}-k_{\perp}^{2}+i \epsilon\right)\left[2\left(x_{1} p^{+}-k^{+}\right)\left(k_{A}^{-}-k^{-}\right)-\left(k_{A, \perp}-k_{\perp}\right)^{2}-m^{2}+i \epsilon\right]} \\
& \simeq \int_{\text {soft } k} d k_{+} \frac{1}{\left(2 k^{+} k^{-}-k_{\perp}^{2}+i \epsilon\right)\left[2 x_{1} p^{+}\left(k_{A}^{-}-k^{-}\right)-\left(k_{A, \perp}-k_{\perp}\right)^{2}-m^{2}+i \epsilon\right]} .
\end{aligned}
$$

As before, $\simeq$ stands for "equality up to power suppressed terms". As one can see, there is no $k^{+}$-pole in the second part of the denominator and we can freely deform the contour in $k^{+}$to avoid the pole in the soft gluon propagator. This takes us out of the soft region for $k$.

In the general situation, Fig. 2 $\mathrm{b}$, we can use a version of the arguments in Ref. 12,24 to show that the soft momenta $k_{i}^{+}$can be rerouted in such a way as to exhibit a lack of a pinch singularity. The essential idea is that one can find a path backwards or forward from one external line of $S$ to another external line of $S$. The loop is completed along lines of $A$, all of which have much larger + momenta than what is typical of soft momenta, and hence there is no pinch.

\section{E. Subtractions}

The subtractions necessary to avoid double counting in the amplitude are treated exactly the same fashion as the ones in Sec. VI of Ref. [12], since the distributional arguments to construct the subtraction terms on a pinch-singular surface $\pi$ presented there are very general in nature and are not limited to the case of diffractive vector meson production that was considered in [12].

The above statement leads to the following asymptotic form of the amplitude

$$
\text { Asy } T=\Sigma_{\Gamma} \operatorname{Asy} \Gamma=A \times H \text {. }
$$

where $\Gamma$ stands for a possible graph for the amplitude $T$.

\section{F. Taylor expansion}

We now obtain the leading term in the hard subgraph, when it is expanded in powers of the small momenta. The arguments used are exactly analogous to the ones used in Sec. VII of Ref. [12] except that we do not have to deal with a $B$ subgraph as was the case in [12]. So we have:

$$
\begin{aligned}
A \times H \simeq & \int d k_{A}^{+} H\left(q, q^{\prime},\left(k_{A}^{+}, 0,0_{\perp}\right),\left(\Delta^{+}-k_{A}^{+}, 0,0_{\perp}\right)\right. \\
& \int d k_{A}^{-} d^{2} k_{A, \perp} A\left(k_{A}, \Delta-k_{A}\right),
\end{aligned}
$$

where $k_{A}$ is the loop momentum joining the $A$ and $H$ subgraphs, and again $\simeq$ means equality up to power-suppressed corrections. Eq. (8) has already a factorized form. However we still have to deal with the extra scalar gluons that may be exchanged between the subgraphs $A$ and $H$; this will be done in the next subsection.

Eq. (8) can be written in the following way: 


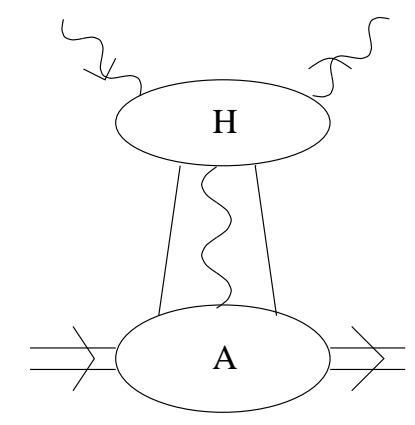

a)
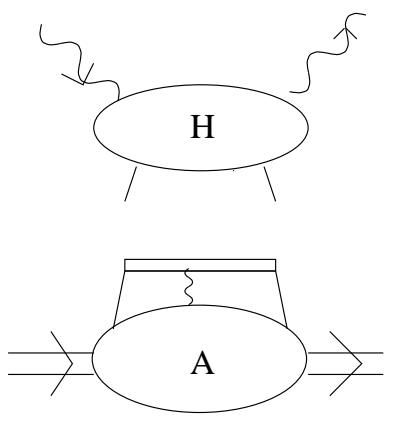

b)

FIG. 4. a) A scalar gluon attaching the collinear subgraph to the hard subgraph $H$ in the unfactorized form. b) Factorized form after application of gauge invariance and Ward-identities. The double line represents the eikonal line to which the scalar gluon attaches.

$$
A \times H \simeq \Sigma_{i} \int d k^{+} C_{i}\left(q, q^{\prime}, k^{+}\right) O_{i}\left(p, p^{\prime}, k^{+}\right),
$$

where the $C_{i}$ are the short distance coefficient functions and the $O_{i}$ are the matrix elements of renormalized light-cone operators.

\section{G. Gauge Invariance}

In order to identify the $O_{i}$ with the parton distributions as defined in [12 (for example), it is necessary to show that all gluons with scalar polarization attaching to the hard graph can be combined into a path-ordered exponential. Fig. 4 shows the example of one scalar

gluon. This was shown in [18,22,23]. Another version of a proof was given in Sec. VII. D of Ref. [12], which relies on very general results recently obtained by Collins [25]. In this way we obtain exactly the same parton distributions as in [12], namely:

$$
\begin{aligned}
& f_{q / p}=\int_{-\infty}^{\infty} \frac{d y^{-}}{4 \pi} e^{-i x_{2} p^{+} y^{-}}\left\langle p\left|T \bar{\psi}\left(0, y^{-}, \mathbf{0}_{\perp}\right) \gamma^{+} \mathcal{P} \psi(0)\right| p^{\prime}\right\rangle \\
& f_{g / p}=-\int_{-\infty}^{\infty} \frac{d y^{-}}{2 \pi} \frac{1}{x_{1} x_{2} p^{+}} e^{-i x_{2} p^{+} y^{-}}\left\langle p\left|T G_{\nu}^{+}\left(0, y^{-}, \mathbf{0}_{\perp}\right) \mathcal{P} G^{\nu+}(0)\right| p^{\prime}\right\rangle .
\end{aligned}
$$

Here, $\mathcal{P}$ represents a path-ordered exponential of the gluon field that makes the operators gauge invariant. The variable $x_{2}$ is the same as in Eq. (3). The evolution equations are the same as in [2,3] and [12].

\section{H. Partons with $k^{+}=0$ : breakpoints and endpoints}

In the factorization theorem Eq. (3), the integral over the fractional momenta includes the points $x_{1}=0$ and $x_{2}=0$. At these points, the hard scattering coefficient for DVCS has a pole, and so we appear to get a logarithmic contribution to the cross section from a region in which one of the lines joining the parton density to the hard scattering subgraphs 


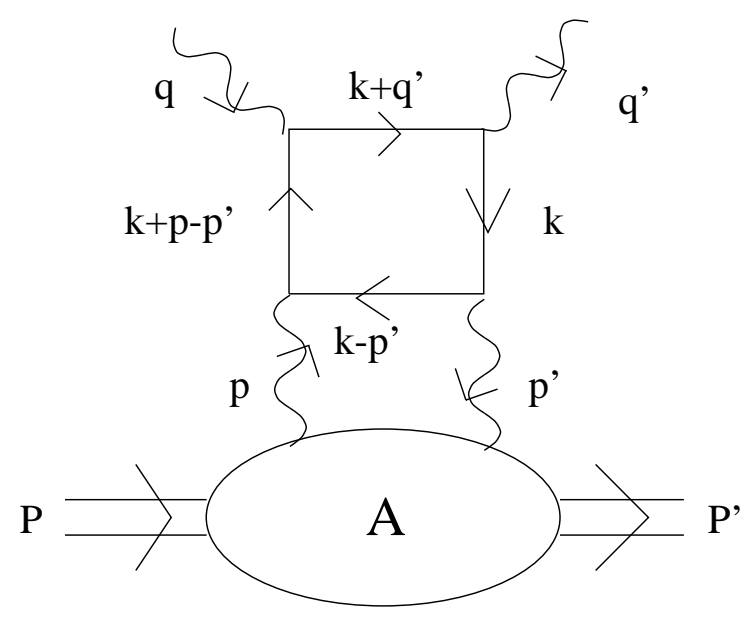

FIG. 5. Particular example of potentially problematic diagram.

is soft instead of collinear. This apparently contradicts our power-counting result that such a region gives a non-leading power. This phenomenon was investigated by Radyushkin [3]. In this section, we will use momentum-space methods to give a general demonstration that the region in question does not give a problem.

First, let us observe that the region of integration over $x_{1}$ in the factorization formula Eq. (3) is $-1+x \leq x_{1} \leq 1$. This is proved by the methods of light-front perturbation theory, by requiring that the intermediate states in Fig. 2 be physically allowed. See Ref. [3,13] for detailed derivations and discussions. The points $x_{1}=0$ and $x_{2}=0$ at which the potential problem arises are what we will call "breakpoints", since they occur in the middle of the range of integration where one of the two lines changes direction.

We continue by examining a particular case, illustrated in Fig. 5, and showing how the argument generalizes. To simplify the example, let us restrict our attention to regions where the subgraph $A$ and the lower three lines $\left(p, p^{\prime}\right.$ and $\left.k-p^{\prime}\right)$ have their momenta collinear to the proton. We will also require the two quark lines, $k+p-p^{\prime}$ and $k$, on the sides of the ladder to have their momenta either collinear to the proton or soft. The example is very similar to one treated by Radyushkin in Ref. [3].

We will also only need the case of the production of a real photon, $q^{\prime 2}=0$, since this is where the problem arises.

The top loop of the graph has the form:

$U=\int d^{4} k \frac{\text { Numerator factors }}{\left(k^{2}-m^{2}+i \epsilon\right)\left[\left(k-p^{\prime}\right)^{2}-m^{2}+i \epsilon\right]\left[\left(k+p-p^{\prime}\right)^{2}-m^{2}+i \epsilon\right]\left[\left(k+q^{\prime}\right)^{2}-m^{2}+i \epsilon\right]}$.

When both $k$ and $k+p-p^{\prime}$ are collinear to $A$, the top line is off-shell by $O\left(Q^{2}\right)$, and it is correct to use the collinear approximation

$$
\frac{1}{\left(k+q^{\prime}\right)^{2}-m^{2}+i \epsilon} \rightarrow \frac{1}{x_{2} Q^{2} / x+i \epsilon}
$$


where $x_{2}=k^{+} / p^{+}$. A corresponding replacement is also to be made in the numerator in $U$. The right-hand side of Eq. (12) exhibits the afore-mentioned pole at $x_{2}=0$. The result of applying the collinear approximation is to give the appropriate contribution to the factorization formula Eq. (3).

The collinear approximation becomes invalid when $k$ becomes soft, i.e., when $x_{2} \rightarrow 0$. We must now demonstrate two facts. The first is that, when $k$ is in a neighborhood of the soft region, the collinear approximation is valid after integration over $k$. The second fact is that the use of the collinear approximation does not give an important contribution from some other region of $k$ which is absent in the original, unapproximated graph. We now examine the integral $U$ in the neighborhood of the soft region for $k$. It has the following form:

$$
\begin{aligned}
U_{\text {soft } k} \simeq & \int_{\text {soft } k} d^{4} k \frac{\text { Numerator factors }}{\left[2 k^{+} k^{-}-k_{\perp}^{2}-m^{2}+i \epsilon\right]\left[2 p^{\prime+}\left(p^{\prime}-k^{-}\right)-\left(p^{\prime}-k\right)_{\perp}^{2}-m^{2}+i \epsilon\right]} \\
& \frac{1}{\left[2\left(p^{+}-p^{\prime+}\right)\left(p^{-}-p^{\prime-}+k^{-}\right)-\left(p-p^{\prime}+k\right)_{\perp}^{2}-m^{2}+i \epsilon\right]\left(\frac{k^{+} Q^{2}}{x p^{+}}-k_{\perp}^{2}-m^{2}+i \epsilon\right)}
\end{aligned}
$$

where we have neglected $k^{+}$in the collinear-to- $A$ lines, $k^{-}$in the collinear-to- $B$ line and $x \neq x_{1}$. We have also ignored the numerator factors, which are an irrelevant complication for our purposes.

According to the power-counting results of Sec. [IIB, which are obtained from [24], the soft region for $k$ gives a power-suppressed contribution. This estimate assumes that all components of $k$ are comparable (in the Breit frame), and is obtained as follows. Let the magnitude of the components of $k$ be $m$. Then the order of magnitude of the soft part of $U$ is a product of factors $1 /\left(m^{5} Q^{3}\right)$ from the denominators, $m^{4}$ from the phase space, and $Q^{2} m^{2}$ from the numerator, for an overall power $m / Q$. This result can be obtained by writing down the largest components in the trace and propagators of Fig. 5 and Eq. (13). Moreover in this region it is correct to replace the fourth propagator in Eq. (13) by its collinear approximation Eq. (12), so that we do not lose the factorization theorem.

However, if the components of $k$ are asymmetric, this estimate no longer holds. In particular if the longitudinal components of $k$ are of order $k^{ \pm} \sim m^{2} / Q$ while the transverse components remain of order $m$, then we get contributions of order $1 / m^{8}$ from the denominators, $m^{6} / Q^{2}$ from the phase space, and $Q^{2} m^{2}$ from the numerators, for a total of $m^{0} Q^{0}$. This shows that the contribution from this region is unsuppressed for large $Q$.

At this point we must appeal to the contour deformation arguments of Ref. [24]. It is only when the integration over $k$ is pinched in the region in question that it needs to be taken into account. In the dangerous region we have $k^{+} k^{-} \ll k_{\perp}^{2}$, so that the only $k^{+}$dependence in Eq. (13) is the pole in the fourth denominator. We can therefore deform $k^{+}$into the complex plain a long way out of the region we are considering, indeed all the way to the collinear-to- $A$ region. Then the collinear approximation is valid so that we can replace the graph by its contribution to the factorization formula.

This contour deformation argument is completely general, as explained in Sec. IIIE of Ref. [24]. Whenever we have a soft momentum with $k^{+} k^{-} \ll k_{\perp}^{2}$, the contour of $k^{+}$can be deformed away from poles in the jet subgraph associated with with the produced photon. Since all the relevant singularities are in the final state, they are all on the same side of the real axis. 
Now that we have established in more detail that the only leading regions are those symbolized in Fig. 2(a), we can apply the collinear approximation as described earlier, and hence we obtain the factorization theorem.

But we still see the following problem. In the factorization theorem, Eq. (3), the parton densities are non-analytic at the breakpoints $x_{1}=0$ and $x_{2}=0$, whereas the coefficient function has a pole at each of these points. Again consider the collinear approximation to Fig. . in the region we were considering. The parton density is non-analytic when $x_{2}=0$, while the coefficient function has a pole there, as is seen from the right-hand-side of Eq. (12). So we cannot literally apply the contour deformation argument.

What we will show is that the parton density is continuous at the breakpoint, so that it can be written as the sum of a function that is analytic at $x_{2}=0$ and a function that has a zero at $x_{2}=0$. The only potential leading twist contribution near the breakpoint is associated with the non-zero analytic term to which the contour deformation argument applies.

To prove this property of the parton density at a breakpoint, consider a general graph for the parton density, as shown in Fig. 6. We have found it convenient to change the labeling of the momentum compared with the previous figure. As always, the $k^{-}$and $\mathbf{k}_{\perp}$ components of $k$ have been short circuited and are integrated over. The $k$-line gives a pole at $k^{-}=\left(k_{\perp}^{2}+m^{2}-i \epsilon\right) / 2 k^{+}=\left(k_{\perp}^{2}+m^{2}-i \epsilon\right) / 2 x_{1} P^{+}$, while the $k+q^{\prime}-q$-line gives a pole at $k^{-}=\left[\left(k+q-q^{\prime}\right)_{\perp}^{2}+m^{2}-i \epsilon\right] / 2\left(k^{+}-\xi P^{+}\right)=\left[\left(k+q-q^{\prime}\right)_{\perp}^{2}+m^{2}-i \epsilon\right] / 2 x_{2} P^{+}$. Here, $\xi$ is the fractional longitudinal momentum transfer $1-P^{\prime^{+}} / P^{+}$. In addition there are poles from the collinear-to- $A$ in the blob. For example if the blob consists of a single line, we have a pole at $k^{-}=P^{-}-\left(k^{2}+m_{\perp}^{2}-i \epsilon\right) / 2\left(1-x_{1}\right) P^{+}$or at $k^{-}=-P^{\prime-}+\left[\left(k+P^{\prime}\right)^{2}+m_{\perp}^{2}-i \epsilon\right) / 2\left(1-\xi+x_{1}\right) P^{+}$.

As we vary $x_{1}$, the $k^{-}$contour can generally be deformed to avoid the poles, so that we have analytic dependence on $x_{1}$. The possible exceptions occur when the $k^{-}$contour is pinched for finite $k^{-}$or when a singularity coincides with the endpoint of the integration at $k^{-}=\infty$. A pinch never occurs; in the general case this is a consequence of the Landau rules. But endpoint singularities occur, and these are precisely at the breakpoints.

For example if $k^{+} \rightarrow 0$, then $k^{+}$can approach 0 from above and below. The pole giving us trouble stems from the $k$-line, all other propagators are unproblematic in this case, since their poles are at finite $k^{-}$. The pole in $k^{-}$approaches $+\infty-i \epsilon$ as one approaches $0^{+}$and $-\infty+i \epsilon$ as one approaches $0^{-}$. This means that the $k^{-}$pole crosses the real axis at infinity. Hence the parton distribution is non-analytic there. Since the singularity is at $\left|k^{-}\right|=\infty$ the other propagators have large denominators, and hence we get a zero for the non-analytic part of integral at the breakpoint. Thus the parton density is continuous at the breakpoint, as claimed. This result enables the factorization formula to be valid in the neighborhoods of the breakpoints. Since the other poles in the $k^{-}$integral are on opposite sides of the real axis, the parton distribution is non-zero at the breakpoints.

Effectively the crossover of the pole occurs when $k$ is in a collinear-to- $B$ region, which we know is power suppressed. This indicates that the argument we have just given generalizes to all graphs.

${ }^{3}$ Radyushkin indicated briefly in Ref. [3] how such a property is to be proved from the $\alpha$ representation for his double distributions. 


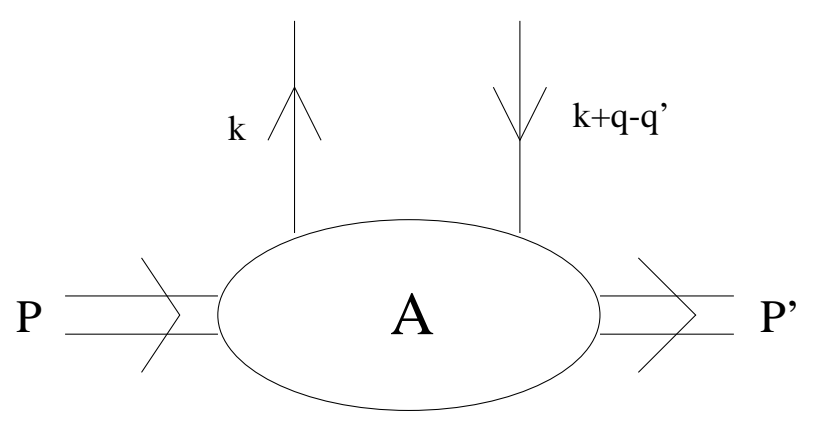

FIG. 6. Parton distribution amplitude.

We also remark on the behavior at the endpoints. Let us look at the case $k^{+} \rightarrow p^{+}$. We find that another of the poles pole runs off to $-\infty$ this time and crosses the real axis there. But now all the other poles are on a single side of the real axis, so that the sole contribution to the parton density comes from the pole at infinity, and hence there is a zero of the parton density at the endpoint.

\section{Completion of Proof}

Using the definitions of the parton distributions and the hard scattering coefficients we finally obtain Eq. (3). Note that the theorem is valid for the production of a real photon which directly goes into the final state and for the production of a time-like photons that decays into a lepton pair.

\section{CONCLUSION}

We have proved the factorization theorem for deeply virtual Compton scattering up to power suppressed terms to all orders in perturbation theory. The form of the theorem is independent of the virtuality of the produced photon.

After this work was complete, we learned from X.-D. Ji that he and Osborne have also constructed a proof of factorization for DVCS [26].

\section{ACKNOWLEDGMENTS}

This work was supported in part by the U.S. Department of Energy under grant number DE-FG02-90ER-40577. We would like to thank L. Frankfurt, X.-D. Ji, A. Radyushkin, and M. Strikman for conversations. 


\section{REFERENCES}

[1] X.-D. Ji, Phys. Rev. D55, 7114 (1997), hep-ph/9609381.

[2] A.V. Radyushkin, Phys. Lett. B380, 417 (1996), hep-ph/9604317.

[3] A.V. Radyushkin, Phys. Rev. D56, 5524 (1997), hep-ph/9704207.

[4] D. Müller, "Restricted conformal invariance in QCD and its predictive power for virtual photon processes", hep-ph/9704406.

[5] X. Ji and J. Osborne, Phys. Rev. D57, 1337 (1998), hep-ph/9707254.

[6] A.V. Belitsky and D. Müller, Phys. Lett. B417, 129 (1998), hep-ph/9709379.

[7] M. Diehl, T. Gousset, B. Pire, and J.P. Ralston, Phys. Lett. B411, 193 (1997), hepph/9706344.

[8] Z. Chen, "Non-Forward and Unequal Mass Virtual Compton Scattering", hepph/9705279.

[9] L. Frankfurt, A. Freund and M. Strikman, "Diffractive Exclusive Photoproduction in DIS at HERA", hep-ph/9710356.

[10] L. Mankiewicz, G. Piller, E. Stein, M. Vättinen and T. Weigl, "NLO Corrections to Deeply-Virtual Compton Scattering", hep-ph/9712251.

[11] J. Bartels and M. Loewe: Z. Phys. C12, 263 (1982);

B. Geyer et al., Z. Phys. C 26, 591 (1985);

T. Braunschweig et al., Z. Phys. C 33, 275 (1986);

T. Braunschweig, B. Geyer, and D. Robaschik, Annalen Phys. (Leipzig) 44, 403 (1987);

F.-M. Dittes et al., Phys. Lett. B 209, 325 (1988);

P. Jain and J.P. Ralston, in: Future Directions in Particle and Nuclear Physics at MultiGeV Hadron Beam Facilities, BNL, March 1993;

I.I. Balitsky and V.M. Braun, Nucl. Phys. B311 (1989) 541;

I.I. Balitsky and A.V. Radyushkin Phys. Lett. B413, 114 (1997), hep-ph/9706410.

J. Blümlein, B. Geyer, and D. Robaschik, Phys. Lett. B406, 161 (1997), hepph/9705264.

[12] J.C. Collins, L. Frankfurt, and M. Strikman, Phys. Rev. D56, 2982 (1997), hep$\mathrm{ph} / 9611433$.

[13] L.L. Frankfurt, A. Freund, V. Guzey, and M. Strikman, Phys. Lett. B418, 345 (1998), hep-ph/9703449.

[14] S.J. Brodsky, L.L. Frankfurt, J.F. Gunion, A.H. Mueller, and M. Strikman, Phys. Rev. D50, 3134 (1994); ibid. Erratum in print, hep-ph/9402283.

[15] M.G. Ryskin, Z. Phys. C 57, 89 (1993).

[16] S.A. Anikin and O.I.Zavialov, Ann. Phys. (N.Y.) 116, 135 (1978).

[17] D. Müller, D. Robaschik, B. Geyer, F.-M. DIttes and J. Hořejši, Fort. Phys. 42, 2 (1994).

[18] J.C. Collins, D.E. Soper, and G. Sterman, Factorization of Hard Processes in QCD, in "Perturbative QCD" (A.H. Mueller, ed.) (World Scientific, Singapore, 1989).

[19] J.C. Collins and G. Sterman, Nucl. Phys. B185, 172 (1981).

[20] S. Libby and G. Sterman, Phys. Rev. D 18, 3252 (1978); 18, 4737 (1978).

[21] G. Sterman, Phys. Rev. D 17, 2773 (1978); 17, 2789 (1978).

[22] A.V. Efremov and A.V. Radyushkin, Teor. Mat. Fiz. 44, 327 (1980) [Eng. transl.: Theor. Math. Phys. 44, 774 (1981)].

[23] J.M.F. Labastida and G. Sterman, Nucl. Phys. B254, 425 (1985). 
[24] J.C. Collins, Phys. Rev. D57, 3051 (1998), hep-ph/9709499.

[25] J.C. Collins, "Ward identities for partially one-particle irreducible Green functions", preprint in preparation.

[26] X.-D. Ji and J. Osborne, "One-Loop Corrections and All Order Factorization In Deeply Virtual Compton Scattering", hep-ph/9801260. 